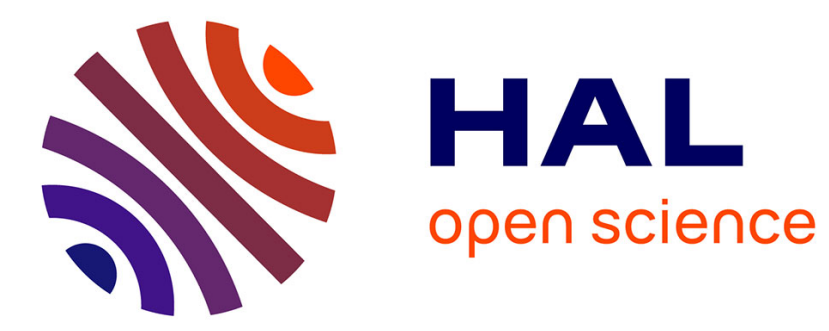

\title{
Valeur perçue des applications mobiles d'aide à l'achat en magasin : une approche hybride par les coûts et les bénéfices
}

Fidan Kurtaliqi, Caroline Lancelot Miltgen, Gaelle Pantin-Sohier

\section{- To cite this version:}

Fidan Kurtaliqi, Caroline Lancelot Miltgen, Gaelle Pantin-Sohier. Valeur perçue des applications mobiles d'aide à l'achat en magasin : une approche hybride par les coûts et les bénéfices. Revue management \& avenir, 2019, 111, pp.123-144. 10.3917/mav.111.0123 . hal-03556282

\section{HAL Id: hal-03556282 \\ https://hal.science/hal-03556282}

Submitted on 3 Feb 2022

HAL is a multi-disciplinary open access archive for the deposit and dissemination of scientific research documents, whether they are published or not. The documents may come from teaching and research institutions in France or abroad, or from public or private research centers.
L'archive ouverte pluridisciplinaire HAL, est destinée au dépôt et à la diffusion de documents scientifiques de niveau recherche, publiés ou non, émanant des établissements d'enseignement et de recherche français ou étrangers, des laboratoires publics ou privés. 


\title{
Fidan Kurtaliqi \\ Université d'Angers - GRANEM \\ fidan.kurtaliqi@gmail.com
}

Fidan Kurtaliqi est professeur assistant à l'ESSCA School of Management. Il a défendu sa thèse de doctorat en 2019. Son travail porte sur l'usage des applications mobiles au point de vente en mobilisant la notion de valeur.

\author{
Caroline Lancelot Miltgen \\ Audencia Business School - Rn'B Lab \\ clancelot@,audencia.com
}

Caroline Lancelot-Miltgen est Professeur en Marketing à Audencia Business School où elle est coresponsable du département marketing, principalement en charge des activités de recherche. Ses travaux s'intéressent à l'impact des technologies sur les usages, avec un focus sur le management des données et la privacy. Elle a été Responsable Scientifique de deux contrats de recherche pour la Commission Européenne sur 'vie privée et systèmes d'identification électronique' (2007) et 'vie privée et management de données personnelles' (2009). Elle est l'auteur de plusieurs articles de recherche scientifiques dans les plus grandes revues françaises et internationales et de chapitres d'ouvrages dans le domaine du Marketing et des Systèmes d'Information, notamment sur la question du management des données clients et de la privacy.

\author{
Gaëlle Pantin-Sohier \\ Université d'Angers - GRANEM \\ gaelle.pantin-sohier@univ-angers.fr
}

Gaëlle Pantin-Sohier concentre ses travaux scientifiques sur le comportement du consommateur dans le laboratoire de recherche GRANEM (groupe de recherche d'Angevin dans les sciences économiques et gestion, un laboratoire de recherche composé de 50 professeurs de recherche et de 40 doctorants) dont elle est la directrice depuis septembre 2015. Elle a défendu sa thèse de doctorat en 2004 et son habilitation de recherches (HDR) en 2014 et dirige plusieurs thèses de doctorat. Sa recherche est consacrée à l'acceptabilité de l'innovation dans le secteur alimentaire et à l'influence des stimuli sensoriels sur le comportement du consommateur, avec plusieurs articles scientifiques publiés dans les journaux nationaux et internationaux. 


\title{
Valeur perçue des applications mobiles d'aide à l'achat en magasin : Une approche hybride par les coûts et les bénéfices.
}

\author{
Fidan KURTALIQI*, Caroline LANCELOT MILTGEN** et Gaëlle PANTIN- \\ SOHIER***
}

* ESSCA School of Management ** Audencia Business School (Audencia Recherche) *** Université d'Angers (GRANEM)

Résumé : Cet article vise à identifier les bénéfices et les coûts liés à l'usage d'applications mobiles d'aide à l'achat en point de vente, en considérant le concept de « valeur perçue ». Si l'usage du smartphone en magasin est devenu courant, celui des applications mobiles interroge la création de valeur pour le consommateur et le distributeur. Jusqu'ici, les recherches se sont concentrées sur l'usage des applications en dehors du magasin, laissant un champ d'exploration pour les usages en point de vente. A travers une étude qualitative exploratoire, ce travail permet d'identifier les sources de création et de destruction de valeur pour des applications mobiles d'aide à l'achat, dans le contexte du point de vente. Les principaux apports théoriques de ce travail sont, d'une part, la mise en évidence de coûts (e.g. dépendance, soi social, perte de plaisir d'achat, etc.) spécifiques aux applications mobiles et à leur usage en magasin et, d'autre part, l'identification d'éléments conditionnels (e.g. degré de personnalisation, type de magasin, etc.) pouvant affecter l'intensité perçue des bénéfices et des coûts. Sur le plan managérial, l'article souligne l'importance des leviers participant à la valeur perçue d'une application mobile et suggère des pistes de réflexion pour réduire la perception des coûts.

Mots clés : valeur perçue, application mobile, coûts-bénéfices, point de vente, distribution.

\begin{abstract}
:
This article aims to identify the benefits and costs related to the use of mobile purchasing assistance applications at the point of sale, considering the concept of "perceived value". While the use of smartphones in stores has become more common, the use of mobile applications raises questions about creating value for consumers and retailers. So far, research has focused on the use of applications outside the store, leaving a field of exploration for in-store uses. Through an exploratory qualitative study, this work identifies the sources of value creation and destruction for mobile purchasing assistance applications, in the context of the retail store. The main theoretical contributions of this work are, on the one hand, the identification of costs (e.g. dependence, social self, loss of purchasing pleasure, etc.) specific to mobile applications and their use in stores and, on the other hand, the identification of conditional elements (e.g. degree of personalization, type of store, etc.) that may affect the perceived intensity of benefits and costs. On the managerial level, the article highlights the importance of the levers involved in the perceived value of a mobile application and gives some recommendations to reduce the perception of costs.
\end{abstract}

Keywords: value in use, mobile application, costs-benefits, point of sale, retail. 


\section{Introduction}

Depuis quelques années, de nombreux distributeurs (e.g. FNAC, Darty, Auchan, Carrefour) ont développé des applications mobiles liées à leur enseigne. Ayant largement contribué au développement du « shopping » mobile (Groß, 2015), ces applications mobiles permettent aux utilisateurs de bénéficier de nombreux services en amont de l'achat (s'informer et commander des produits, faire des listes d'achats, etc.). En magasin, plus d'un client sur deux utilise le smartphone pour comparer les prix (40\%), contacter un ami ou la famille pour un conseil $(40 \%)$ et pour prendre des photos du produit $(36 \%)^{1}$. Pour autant, ces usages ne nécessitent pas nécessairement le recours à une application mobile d'enseigne. Les navigateurs, disponibles sur les smartphones, peuvent suffisent pour comparer des prix de produits, par exemple. Dès lors, l'usage et la création de valeur de ces applications d'aide à l'achat au point de vente, pose question.

Si la majorité des consommateurs a d'ores et déjà adopté ces outils technologiques, mieux identifier leur valeur pour le client est primordial, dans la mesure où celle-ci détermine l'usage à plus long terme de ce type de service. La littérature révèle que les recherches sur la valeur perçue des applications mobiles n'en sont qu'à leur début.

Les leviers de création et de destruction de valeur, ainsi que leur impact sur la valeur globale, ont été mis en lumière dans le domaine des SST (self-service technologies) et des SSIT (selfservice information technologies) (Feenstra et Glérant-Glikson, 2017; Lapassouse-Madrid, et Vlad, 2016; Mencarelli et Rivière, 2014). Jusqu'à présent, les travaux concernant les applications mobiles se sont davantage centrés sur la question de leur adoption (Newman et al., 2017). Ces dernières années, d'autres recherches ont émergées en adoptant une approche multidimensionnelle de la valeur perçue des applications mobiles (Gonzalez et al., 2012; Gummerus et Pihlström, 2011). Cependant, ces travaux n'ont pas véritablement apporté d'éclairage quant à la diversité des coûts possiblement liés à l'usage de ces applications, notamment du fait que cette approche multidimensionnelle a pour objectif unique de présenter les composantes de la valeur (Rivière et Mencarelli, 2012). Kumar et Reinartz (2016) soulignent que la digitalisation a fait émerger de nouveaux « coûts » liés à l'exposition publicitaire ainsi qu'à la divulgation des données personnelles. Par ailleurs, la gratuité des services mobiles, amène à s'intéresser à d'autres coûts que les coûts traditionnels traités à travers le prisme monétaire, ce que les recherches actuelles n'ont pas encore suffisamment pris en compte (Kumar et Reinartz, 2016).

Même si la littérature dans le domaine des SST souligne de nombreux coûts perçus à l'usage de ces outils, il est risqué de considérer que ces coûts sont directement transposables aux applications mobiles. Selon Newman et al. (2017) et Collier et al. (2014), les consommateurs n'ont pas un comportement uniforme envers toutes les SST. Or, si les applications mobiles peuvent être considérées comme des SST à part entière, du fait qu'elles sont utilisées par des terminaux appartenant aux clients (smartphone), elles présentant des caractéristiques bien différentes (e.g. connexion internet) des autres SST (e.g. caisses automatiques).

Inspirés des travaux réalisés dans le domaine des SST par Mencarelli et Rivière, (2014), nous souhaitons comprendre les usages des applications mobiles en magasin dans l'objectif d'identifier les bénéfices et/ou les coûts perçus par leurs utilisateurs. Nous suivons ainsi Robertson et al. (2016) qui recommandent de mener des recherches sur différents types de SST.

\footnotetext{
${ }^{1}$ GFK (2015), Consumers' activities with mobile phones in stores.
} 
L'approche hybride de la valeur, initiée par Aurier et al. (2004), est mobilisée parce qu'elle comporte à la fois les avantages de la vision unidimensionnelle et ceux de la vision multidimensionnelle.

Plus précisément, ce travail a pour ambition d'étudier les différences de perception de la valeur selon la nature de l'application. Sheth et al. (1991) et Wang et al. (2013) ont montré l'importance du contexte dans la perception de la valeur d'un objet/service. Notre étude se concentrera sur deux types d'applications mobiles : les applications pull (permettant un contrôle total de l'application et des notifications), et les applications push (qui ne permettent aucun contrôle des notifications). A travers ces deux types d'applications, nous comparons en quoi le fait d'avoir ou non le contrôle sur la manière dont fonctionne l'application, modifie la perception de la valeur. Nous avons également demandé aux répondants quelle serait l'application idéale pour eux, afin d'identifier quels sont les bénéfices plébiscités et les coûts que les utilisateurs cherchent à minimiser. Enfin, nous avons interrogés les participants sur leur perception des applications de M-commerce, dans l'objectif de comparer les résultats avec les applications utilisées en point de vente physique.

Pour résumer, cet article cherche à répondre aux deux questions de recherche suivantes : quelles sont les sources de création (i.e. bénéfices) et/ou de destruction (i.e. coûts) de valeur pour le client lorsqu'il utilise une application mobile d'aide à l'achat en magasin ? Est-ce que les individus perçoivent les mêmes sources de valeur selon la nature de l'application et le contexte d'utilisation?

Après avoir proposé une synthèse de la littérature, nous exposons les apports de l'étude des applications mobiles à travers le prisme de la valeur. Les résultats de l'étude qualitative permettent de dégager des contributions théoriques et managériales discutées dans la conclusion.

\section{Les applications mobiles, un champ de recherche en pleine ébullition}

L'utilisation quotidienne du smartphone a profondément modifié les comportements d'achat des consommateurs. Chercher, comparer et acheter des produits et/ou des services en ligne où que l'on soit età n'importe quelle heure est dorénavant chose aisée (Groß, 2015). Face à ces bouleversements créés par le digital, le commerce physique a décidé de suivre cette tendance à la digitalisation en développant les SST. $80 \%^{2}$ des distributeurs ont fait du « shopping mobile » une priorité.

Faisant partie des SST, l'application mobile a fait l'objet de nombreuses études visant à mieux comprendre les différents stades d'appropriation de cet outil depuis l'adoption jusqu'à l'usage voire le post-usage (Newman et al., 2017). Le majorité des travaux se sont focalisés sur l'adoption des applications mobiles (Nysveen et al., 2005) en utilisant, en particulier, le modèle TAM (Davis, 1989). Ces travaux ont ensuite été complétés en intégrant des dimensions de satisfaction (Revels et al., 2010), mais aussi des approches par la valeur, dans l'objectif de mieux identifier les facteurs déterminant l'intention d'usage (Kleijnen et al., 2007). Ces modèles d'adoption ont également été transposés au marketing mobile (Kang et al., 2015; Merisavo et al., 2007). Ces analyses ont mis en lumière l'impact de la facilité d'utilisation et de la fréquence d'usage sur les achats via le mobile (Newman et al., 2017), mais aussi l'importance du rôle de la valeur conditionnelle (H.-Y. Wang et al., 2013) sur l'usage de l'application. En, effet, cette dernière (i.e. la valeur conditionnelle) influence de manière considérable les variables fonctionnelle, sociale, épistémique et émotionnelle. De plus, des corrélations positives

\footnotetext{
${ }^{2}$ Mobile Marketing Association France. (2017) : Observatoire du commerce mobile. $2^{\text {ème }}$ édition.
} 
ont été mises en évidence entre le caractère ubiquitaire (Tojib et Tsarenko, 2012), la qualité du service mobile et la satisfaction client.

Toutefois, il faut noter que ces travaux s'intéressent généralement aux achats en ligne. Or les achats en ligne via l'application, n'incitent pas systématiquement les clients à se rendre par la suite au point de vente physique (Newman et al., 2017). Cela démontre que les applications de M-commerce ne remplissent pas forcément un rôle de connexion entre le commerce virtuel et physique, mais peuvent être davantage une source de cannibalisation. Dès lors, inciter leurs clients à recourir à des applications mobiles d'aide à l'achat constitue, pour les distributeurs, un enjeu majeur, à l'heure où les clients utilisent toujours plus de sources d'informations numériques pour se renseigner sur les produits/services qu'ils souhaitent acquérir (Kallweit et al., 2014). Si plus d'un client sur deux utilise son smartphone en magasin pour comparer les prix, l'intérêt pour le distributeur est de faire en sorte que celui-ci compare les produits du magasin via l'application de l'enseigne et non via un navigateur internet, permettant de consulter les prix de la concurrence.

Les travaux portant sur l'usage des applications d'aide à l'achat en magasin restent encore marginaux, du fait qu'elles sont des SST émergentes (Newman et al., 2017). Par ailleurs, les études relatives au M-commerce peuvent difficilement expliquer les usages des applications en magasin et ne sont donc pas duplicables en l'état. Ces applications d'aide à l'achat revêtent des caractéristiques bien spécifiques nécessitant de les étudier indépendamment des autres SST. En effet, la majorité des applications étant installées sur les smartphones des clients, des questions spécifiques se posent comme l'atteinte à la vie privée, le suivi des clients en magasin, les effets sur les interactions avec les vendeurs du point de vente, etc.

\section{Le concept de valeur}

La valeur s'est montrée incontournable dans son usage pour mieux comprendre l'évaluation d'un objet par un individu. En marketing, trois approches permettent traditionnellement d'aborder la valeur perçue : l'approche unidimensionnelle, multidimensionnelle et hybride. La première considère la valeur comme la résultante d'un raisonnement cognitif ayant recours au ratio sacrifices-bénéfices (Dodds, Monroe, et Grewal, 1991). Les travaux mobilisant cette approche ont apporté des éclairages sur l'effet du prix et de la marque sur la perception de la qualité et de la valeur du produit (Dodds et Monroe, 1985; Zeithaml, 1988). Malgré le nombre important de travaux mobilisant le ratio qualité-sacrifice pour caractériser l'achat de produits (Agarwal et Teas, 2001; Chang et Wildt, 1994; Sweeney, Soutar, et Johnson, 1999) et de services (Brady et Robertson, 1999; Bolton et Drew, 1991), cette approche a fait l'objet de plusieurs critiques conceptuelles. On reproche notamment à celle-ci d'avoir une vision réductrice de la valeur, ignorant les aspects émotionnels (De Ruyter, Wetzels, Lemmink, et Mattson, 1997; Mathwick, Malhotra, et Rigdon, 2001; Sweeney et Soutar, 2001; Zaune). Sweeney et Soutar (2001, p. 2007) soulignent qu'il est nécessaire d'avoir un modèle de mesure plus sophistiqué pour comprendre comment les consommateurs valorisent les produits et les services.

De là, a émergé une deuxième vision de la valeur dite multidimensionnelle. Elle trouve son origine dans les premières définitions apportées par Hirschman et Holbrook sur la consommation expérientielle qui développent les dimension relative, émotionnelle (Hirschman et Holbrook, 1982, 1982) et contextuelle (Holbrook et Corfman, 1985) de la consommation. A la différence de l'approche unidimensionnelle qui consiste à évaluer une valeur globale, l'approche multidimensionnelle s'attache à identifier les composantes de la valeur. Elle s'inscrit par conséquent dans une approche analytique de la valeur (Rivière et Mencarelli, 2012). Si cette vision apporte une richesse dans la compréhension des composantes de la valeur, elle 
n'explique pas pour autant le rôle de chacune de ses composantes dans la construction de la valeur globale. Face aux limites de ces deux premières approches, une approche dite « mixte " ou « hybride » a émergé (Rivière et Mencarelli, 2012). Albert Wenben Lai (1995) fut l'un des premiers à plaider en faveur de cette approche hybride consistant à rapprocher la vision unidimensionnelle, issue de l'étude de la valeur d'achat, et la vision multidimensionnelle, provenant des recherches sur la valeur de consommation. Aurier et al. (2004), ont adopté cette dernière approche mesurant ainsi la valeur globale dans le contexte du cinéma.

Dans le cadre des applications mobiles, plusieurs travaux ont mobilisé l'approche multidimensionnelle pour déterminer l'adoption (Kim et Oh, 2011; Kim et Han, 2011), l'utilisation (Wang et al., 2013) et la poursuite de l'utilisation des services mobiles (Ozturk et al., 2016). D'autres recherches utilisent l'approche multidimensionnelle de la valeur dans l'objectif d'établir des liens entre la valeur conditionnelle et la valeur perçue (Gummerus et Pihlström, 2011). Gonzalez et al. (2012) identifient, pour leur part, les sources de valeur ayant un impact sur la valeur globale de consommation d'une application, son intention d'utilisation, ainsi que l'impact sur le bouche à oreille et la relation à la marque.

L'approche hybride de la valeur apporte une meilleure compréhension de la valeur globale dans le cadre des services (Aurier et al., 2004; Ruiz, Gremler, Washburn, et Carrión, 2008). Mencarelli et Rivière (2014) ont utilisé cette approche dans l'étude des SST, permettant ainsi de faire émerger des dimensions de bénéfices et de sacrifices contribuant à la construction d'une valeur globale perçue chez les consommateurs. Dans cette même veine, d'autres travaux ont identifié les leviers de création et de destruction de valeur chez les consommateurs (LapassouseMadrid et Vlad, 2016 ; Feenstra et Glérant-Glikson, 2017) et les managers (Lapassouse-Madrid et Vlad, 2016), et la contribution de ces différents leviers à la valeur globale perçue des SST. Pour ce qui est des applications mobiles, Kleijnen et al. (2007) proposent un modèle théorique basé sur le rapport de trois bénéfices (contrôle de l'application, compatibilité et gain de temps) et deux coûts (risques et effort cognitif) permettant d'expliquer l'adoption d'une application mobile.

A la lumière de ces études, nous pouvons définir les points clés à partir desquels notre travail s'oriente. En premier lieu, nous devons souligner que les travaux réalisés sur les SST, en mobilisant l'approche hybride de la valeur, sont difficilement transposables aux applications mobiles. En effet, les spécificités de chaque SST peuvent influencer la perception de la valeur de l'objet/service (Holbrook et Corfman, 1985). En second lieu, il convient de prendre en compte de nouveaux coûts ayant émergés avec le digital. Ainsi, l'usage des données personnelles et l'exposition aux publicités doivent faire l'objet d'une plus grande considération dans la mesure de la valeur (Kumar et Reinartz, 2016). Bien que pionniers, les travaux de Kleijnen et al. (2007) ne prennent pas en compte ces phénomènes du fait de leur émergence récente. Enfin, le contexte peut avoir un rôle déterminant dans la perception de la valeur. Sheth et al. (1991) parlent alors de « valeur conditionnelle » qui occupe la même place que les autres dimensions de la valeur. Wang et al. (2013) ont ainsi démontré l'influence de la valeur conditionnelle sur la valeur fonctionnelle, sociale, émotionnelle et épistémique liée à l'utilisation d'une application mobile.

En résumé, compte tenu de l'importance du contexte, l'étude de la valeur de ces outils dans le contexte du point de vente physique présente un intérêt majeur. Le choix de l'approche hybride de la valeur se justifie par la richesse que celle-ci apporte dans la compréhension de la valeur globale (Mencarelli et Rivière, 2014), en identifiant à la fois les bénéfices et les coûts liés à l'usage de l'application. Enfin, si toutes les SST ne sont pas perçues de manière identique 
(Newman et al., 2017), il est légitime de se poser la question de l'influence de la nature de l'application sur l'identification des sources de valeur.

\section{Méthodologie}

Une étude exploratoire comprenant trois focus groups, de 45 à 60 minutes chacun, a été menée sur la base d'un guide d'animation comprenant six thématiques : (1) utilisation du smartphone au quotidien, (2) dernier achat (réalisé avec le mobile), (3) application préférée (pour s'informer, comparer et/ou acheter via le mobile), (4) application pull (application d'enseigne avec paiement via le mobile), (5) application push (système i-Beacon avec envoi de notifications) et (6) application idéale. Les trois premières thématiques ont pour objectif de générer les sources de valeur, liées à l'expérience d'usage actuel d'une ou plusieurs applications mobiles. Les deux thématiques suivantes visent, dans le cadre de techniques projectives, à immerger les interviewés dans l'utilisation d'applications mobiles conçues pour un usage en point de vente. Deux vidéos d'applications mobiles répondant à ces thématiques ont été diffusées afin que les sujets puissent s'imaginer utiliser ces applications (projection d'usage). La première vidéo porte sur une application de l'enseigne IKEA. On y voir une cliente utiliser l'application dans le magasin. Elle reçoit régulièrement des notifications sur les offres du magasin tout au long de son parcours d'achat. Ces notifications sont envoyées en mode «Push» (i.e. sans qu'elle puisse les contrôler). La deuxième vidéo se déroule dans une librairie. On y voir également un homme utiliser l'application mobile. Celui-ci effectue plusieurs opérations comme le scan des étiquettes des produits pour s'informer mais aussi le paiement des articles sans passer par la caisse. L'application est cette fois contrôlable au niveau des notifications. La thématique 'application idéale' a également pour finalité de générer des sources de valeur en amenant le participant à décrire l'application qui serait, selon lui, idéale. $\mathrm{Au}$ total, 21 individus, détenteurs d'un smartphone, utilisateurs réguliers d'applications mobiles, fréquentant les points de vente physiques et aux profils variés (étudiants, employés, techniciens agents de maîtrise et cadres) ont participé à l'étude. En nous basant sur les recommandations faites par Mukamurera et al. (2006) nous avons stoppé la collecte après atteinte du phénomène de saturation des données. La totalité des échanges a fait l'objet d'un enregistrement audio et d'une retranscription intégrale. L'analyse s'est faite à l'aide du logiciel Nvivo, en suivant la méthodologie de Spiggle (1994) qui apporte des éclairages sur la catégorisation des propos (dans notre cas par coûts et bénéfices), sur la dimensionalisation et l'intégration des propos. Ces opérations ont permis de faire ressortir les différentes dimensions de bénéfices et de coûts.

\section{Les résultats}

Les résultats de la thématique "utilisation du smartphone au quotidien » ont été écartés en raison de leur caractère trop général. Portant sur les achats en ligne via des applications, les thématiques "dernier achat» et "application préférée », ont été fusionnées. Nous avons souhaité garder ces résultats pour pouvoir les comparer avec ceux liés aux applications utilisées en point de vente.

\subsection{Les bénéfices d'usage}

\subsubsection{Bénéfices communs à tous les types d'applications}

L'analyse des résultats fait ressortir deux bénéfices communs à l'ensemble des applications (hormis l'application push). Il s'agit des bénéfices de praticité et d'efficacité.

La praticité a fait l'objet de nombreux travaux antérieurs (Lai, 1995; Sheth et al., 1991; Babin et al., 1994). Pour les quatre applications, les propos liés à la praticité font référence à certaines fonctionnalités comme la possibilité de commander ("Moi je trouve ça plus pratique 》(F41)) de payer aisément (" Avouez que c'est trop simple de payer par téléphone ? ... Et y'a pas à sortir sa carte bleue et payer et voir le montant qui se débite! »), de s'informer ("Comparaison des prix! »(F21)), de scanner un produit, et enfin, d'avoir ses cartes de fidélité sur soi («Les

\footnotetext{
${ }^{3} \mathrm{~F}$ pour Femme, H pour Homme, chiffre correspondant à,l'âge e.g. 41 pour 41 ans
} 
cartes de fidélité aussi, maintenant c'est sur le téléphone » (F46)), etc. Pour les utilisateurs, une application pratique permet l'accès à un large choix de produits et à la possibilité de s'informer de manière détaillée sur ceux-ci. De même, la praticité réside également dans le fait de pouvoir consulter les avis sur les produits, de comparer les prix, de recevoir et de gérer les offres de réduction.

Quant à l'efficacité, elle est généralement intégrée dans le concept global de fonctionnalité (Lai, 1995). Elle permet au consommateur d'aller au-delà des possibilités d'actions, c'est à dire de répondre à des objectifs économiques et pratiques comme le gain de temps ("Elle nous ferait gagner du temps. " (F18)) [application idéale] et les économies d'argent ("Oui parce que ça va te faire faire des économies. "(F46)) [application pull]. Pour les achats en ligne, une application efficiente permet avant tout de trouver des produits peu chers ( $M$ Moi, Wish! Ce n'est pas cher! » (F21)).

\subsubsection{Bénéfices spécifiques au type d'application étudié}

Si les bénéfices d'efficacité et de praticité sont génériques, d'autres bénéfices plus spécifiques ressortent pour certains types d'application. C'est le cas du bénéfice organisationnel, qui fait référence à la capacité de l'application à permettre à l'utilisateur d'organiser ses achats. On peut faire une analogie avec le comportement web-to-store, permettant de préparer l'achat en ligne avant le passage au point de vente (Bouzid et Vanheems, 2014). Ainsi, on retrouve ce bénéfice pour l'application de M-commerce, qui joue un rôle prépondérant dans la préparation des achats (« Si on sait qu'on a un achat prochainement à faire, on peut aller en repérage sur son téléphone si on est dans le bus. » (F21)). L'application idéale devrait permettre de consulter les produits chez soi et d'établir une liste de courses pour préparer l'achat. (" ... Mais avant chez nous, ça peut être pratique d'avoir une application, de regarder, ça, ça pourrait être intéressant. Limite faire une petite liste où on pourrait cocher, filtrer. Ça, ça, ça m'intéresse, du coup j'arrive dans le magasin je sais direct, ah il me manque ça, je l'ai vu. » (F19))

Le bénéfice spécifique du contrôle est relevé pour l'application pull. Il se réfère à la possibilité pour l'utilisateur d'avoir la maîtrise de l'application à tout moment ("Quand tu veux ouais... » (F23). Cela implique entre autres la possibilité de stopper l'utilisation de l'application à tout moment et de gérer les notifications mobiles pendant le parcours d'achat ( «... c'est bien parce que tu peux choisir sur quoi tu veux avoir des infos... " (H22). Ceci est particulièrement rassurant pour l'utilisateur puisqu'il n'a pas l'impression d'être dirigé dans son acte d'achat (" Le fait de maîtriser, oui, ça rassure » (F46))

\subsubsection{Une forte dimension sociale...}

Au-delà de ses capacités pratiques, l'application mobile permet également de créer des liens. Généralement, on parle de valeur sociale, qui se réfère à la capacité du produit à associer l'individu à une classe sociale (Lai, 1995, p.383). Le terme " social » est assez généraliste et comporte de nombreuses acceptions. Dans notre cas de figure, la dimension désigne l'habilité de l'application à permettre au client de rester en contact avec autrui et/ou de susciter une communication avec autrui. L'application idéale par exemple, joue un rôle de socialisation. La satisfaction générée par son utilisation crée des liens sociaux lorsque l'individu présente son application à son entourage ("On leur expliquerait que c'est bien et qu'il faut l'avoir. On pourrait expliquer les avantages. "(F19)), ("Après si on est satisfait, on serait content de montrer une nouvelle application géniale à l'autre. " (F18)).

L'application participe aussi à améliorer la position de l'individu en lui conférant une meilleure image dans la société. Nous évoquerons ici le soi social qui représente "la totalité des pensées et sentiments individuels qui font référence à soi-même en tant qu'objet" (Rosenberg, 1979, p. 
7). Dans le soi social il y a le réel et le rêvé qui correspondent à : ce que les autres pensent de moi et ce que je voudrais que les autres pensent de moi (Vernette, 2008). Pour l'application pull, cela correspond à un sentiment de modernité ("T'es in, moderne " (F46)). Utiliser l'application idéale permet de se sentir en avance sur son temps ("Moi je me sentirais surtout en avance. Un peu lanceur du projet, de l'innovation... » (H19)). Il est important de souligner que le sentiment de fierté ressenti ne s'apparente pas à une autoglorification ou à une démonstration de richesse mais davantage à la modernité et au fait de vivre avec son temps (" Surtout en avance, pas plus riche, pas en train de me la péter... vraiment en avance sur son temps. » (F19)).

\subsection{4 ...animée par l'amusement et le besoin de contrôle}

Une application mobile peut également divertir l'utilisateur dans la mesure où elle possède cette « capacité à englober les besoins de joie, d'amusement, de plaisir ou de distraire du travail et de l'anxiété » (Lai, 1995, p.384). L'application de M-commerce contribue à rendre l'acte d'achat plus hédonique ("Ah ouais je prends bien du plaisir! ... quand je passe la commande je fais " ahhh » ... en fait t'as l'excitation d'acheter! 》(H22)). L'usage peut aussi se révéler plus amusant ("C'est ludique c'est quelque chose qui est sympa quoi... je ne peux pas t'expliquer le mot ludique mais oui ce serait ludique ..." (H22,)) notamment pour l'application pull, grâce aux options et aux informations disponibles sur les produits ( $C$ 'est amusant à utiliser, tu cliques partout, tu as des infos sur les produits » (F18)).

Au-delà de l'amusement, les clients ont besoin d'une application qu'ils contrôlent, qui inspire confiance et ne réserve pas de surprises. Cela implique que le consommateur connaît la destination de ses données mais également que l'application (dans ce cas idéale) ne va pas perturber celui-ci par des messages intempestifs pendant l'acte d'achat (" Et moi j'ajouterai, une appli honnête, qui ne réserve pas de mauvaises surprises. C'est-à-dire, euh, si tu te retrouves noyé sous les messages alors que tu n'avais jamais demandé ça, ça m'embête » (H48)).

\subsection{Les coûts d'usage}

\subsubsection{L'invasion des applications mobiles et les dangers liés à la vie privée}

S'il y a un coût commun aux quatre types d'applications, c'est celui liée à l'invasion des applications qui se caractérise de deux manières. En premier lieu, il se manifeste à travers la réception de notifications (publicités mobiles). Ce premier élément fait référence au concept d'intrusivité (intrusiveness) qui a été mis en exergue dans les médias traditionnels à travers la publicité (Vespe, 1997), et par la suite défini comme le degré par lequel les publicités dans les médias interrompent un flux éditorial (Ha, 1996, p.77). Cette définition est transposable ici du fait qu'une publicité sur mobile représente également une interruption. Pour l'application Mcommerce, l'invasion se manifeste après les achats, avec la réception de publicités, ("Bah c'est chiant pour les mails par contre, les pubs et tout »(F41)). Sur l'application push, ce sentiment prend de l'ampleur avec l'envoi de notifications, ("C'est intrusif! » (H48)). Malgré le contrôle total des notifications, il y a toujours une impression d'invasion pour l'application pull (" Après, oui tu reçois des pubs » (H48)), (" Mais voilà, oui c'est le risque, c'est d'être envahi... »(F46)). L'invasion se manifeste également à travers la place que l'application occupe pendant le processus d'achat. L'application peut paraître invasive parce qu'elle est l'intermédiaire entre le client et le produit. Sur l'application idéale, c'est à travers la place que l'application peut occuper pendant l'achat, que ce coût émerge ("En fait faut pas que ça prenne trop de place quoi! »(F23)), qui donne une impression de voir sa vie dirigée par un outil (" Ouais faut pas que ça dirige notre vie! Du moins ma vie... »(H23)). 
Un danger commun à l'application de M-commerce, pull et push, est celui relatif aux données personnelles. Ce point se réfère à l'atteinte à la vie privée et plus précisément aux préoccupations des individus quant à la manière dont les informations les concernant sont communiquées aux autres (Lancelot-Miltgen et Lemoine, 2015). Pour les achats en ligne via l'application, les interviewés sont conscients de la récolte de données personnelles et savent que les entreprises empiètent sur leur vie privée ("Et puis ça rentre dans notre vie privée aussi. » $(F(2) 19))^{4}$. Pour l'application push, l'envoi de notifications par exemple, accentue le sentiment qu'on pénètre chez soi ("Ça fait bizarre en fait, c'est comme si quelqu'un entrait chez moi ... » (H22)). Concernant l'application pull, le fait que l'entreprise puisse étudier les comportements des consommateurs pendant le parcours d'achat en magasin est difficilement acceptable "Parce que le magasin n'a pas à savoir combien de temps je passe en rayon." $(F(2) 19)$ ) et cela revient à une surveillance des personnes ("Ça fait un peu, big brother is watching you, 1984. Mais vraiment. » (F(2)18)).

\subsubsection{Le sacrifice des relations humaines et de l'éthique}

Si les relations humaines (i.e. coût relationnel) ne sont pas concernées pour l'application de M-commerce, il en est tout autrement pour les applications utilisées en point de vente. L'utilisation d'une application mobile peut être une barrière au contact client-vendeur. L'application idéale doit préserver le contact avec le vendeur (« On ne veut pas que ça remplace le vendeur! »(F23)). Le conseil du vendeur semble être indispensable aux yeux des interviewés, parce que le contact humain rend l'avis plus crédible. C'est le cas pour l'application push ("Après ça ne remplace pas un vieux vendeur qui vient dire " Eh voilà ily $a c ̧ a » . . . »(F 23))$. De manière générale, pour l'application pull, on relève un fort sentiment de sacrifice des relations client-vendeur (" ... ça enlève le côté humain des personnes là, carrément, je trouve... ça ferait vachement de passages dans le magasin ça serait horrible sans que personne ne passe par la caisse » (H22)).

D'après les propos, le développement des services mobiles aurait pour conséquence prévisible la suppression des emplois des hôtes et hôtesses de caisse. Pour l'application pull par exemple, cela soulève des questions éthiques sur le devenir du métier ("Si demain, tout le monde utilise ça, on n'a plus de caissière » (H48)). Ces questions éthiques peuvent conduire au boycott des $\mathrm{SST}$, ("Moi, je suis très réfractaire parce que déjà j'utilise que les caissières parce que je tiens à ce que le peu d'emploi, et en temps partiel qu'elles ont, donc je n'utilise jamais donc je fais la queue.» (F55)). On relève les mêmes propos pour l'application idéale, ("Moi je garde le vendeur, parce que ça crée des emplois... »(H22)).

En dernier lieu, l'usage d'une application mobile pull sacrifie non seulement les relations humaines, les emplois des caissièr(s)es, mais participe aussi, d'après certains propos, à ternir l'image même de l'utilisateur. En effet, en utilisant l'application en magasin, certains clients craignent de véhiculer une image négative et de se sentir associables ("Alors j'ai un mot parfait qui conviendrait parfaitement, désocialisation » (F23)) ou déconnectés de la vie sociale ("Qui se renferment sur elles-mêmes... ! »(H23)). Le paiement via l'application sans passer par la caisse donne le sentiment de ne pas respecter ses obligations ("Tu pars comme un voleur!" (H23)).

\subsubsection{Des coûts spécifiques à la nature des applications}

Dans cette partie liée aux coûts spécifiques, on peut souligner, en premier lieu, la perte de contrôle qui ressort de l'application push. Les interviewés ont l'impression que l'application

\footnotetext{
${ }^{4}$ Femme 2, 19 ans
} 
dirige leur parcours d'achat ("Allez au rayon A après tu vas le suivre, 10\% là-dessus, allez boire un café, il y a $5 \%$, allez ... »(F40)). Dès lors, on passe d'une situation de liberté d'achat à une situation où on force à l'achat ("C'est le téléphone qui te dit ce que tu dois faire..." (F35)). Ceci est perçu comme une contrainte ("J'aurais l'impression d'être contrainte à faire quelque chose. " (F(3)19) et une perte d'indépendance ("Ça nous incite à ne plus réfléchir. " (F(1)19)).

Dans la continuité de la perte de contrôle, on relève un coût émotionnel résultant de l'usage de l'application push. Rappelons que la dimension fait référence à la capacité de l'objet à susciter des émotions ou des états affectifs (Sheth, Newman, et Gross, 1991, p.161). Dans notre cas, ces états sont générés, en premier lieu, par les notifications. Elles provoqueraient l'agacement à cause des envois intempestifs ( "C'est vrai que ça peut vite t'agacer » (F21)), ("Tu te dis, je n'y vais pas parce qu'ils vont encore m'agacer avec leur machin »(F41)). En second lieu, il ressort un problème de surveillance. Se savoir surveillé à travers l'application effraie. ("Là par contre je trouve que ça fait peur! " (H22)).

L'utilisation d'une application mobile requiert la participation active de son utilisateur. Si l'implication cognitive demandée est perçue comme intense, elle provoque un coût lié à l'effort ((«Moi, c'est plutôt un effort d'utiliser une appli. En fait, ça me fatigue. "(F(2)18)) [application idéale].

Nous avons évoqué précédemment le sacrifice des relations client-vendeur, pouvant être provoqué par l'usage des applications mobiles en magasin. Ce sacrifice pourrait potentiellement s'étendre à la relation client-produit. La dimension « perte de plaisir d'achat » a été relevée pour l'application idéale. Elle représente l'effet inverse de la dimension hédonique. Utiliser l'application serait un sacrifice puisque cette utilisation aurait pour effet de dénaturer l'acte d'achat ("Pour avoir le temps de marcher, de regarder un peu partout, de faire tous les magasins même ceux qui me n'intéressent pas juste histoire de flâner en fait et moi c'est ça qu'il ne faut pas dénaturer, le temps de flâner!"(H22)). Selon les répondants, l'achat dit hédonique ne doit en aucun cas, être perturbé par une application (" ... quand t'es dans le magasin, tu regardes les articles, tu regardes les rayons, t'es là pour regarder ce que tu peux acheter, tu ne regardes pas ton téléphone » (F41))

En dernier lieu, une dimension liée à la dépendance est relevée pour l'application push. D'après les propos, les applications contribuent fortement à rendre les utilisateurs dépendants ("Non mais ce que je veux dire c'est que sans le téléphone, on ne sait plus rien faire du coup. On dépend du téléphone. »(F(2)19)). Ceci pose un réel problème pour les utilisateurs puisqu'ils affirment ne plus être capables de faire sans par la suite ("Bah, oui, on se sent dépendant. » $(F(2) 18)$ ). Il y a par conséquent, grâce au confort et à la rapidité de l'application, un risque de non-incitation à l'action et à la réflexion ("Tu n'es plus capable de faire ton chemin sans Google Maps. » (F(2)19)).

\subsection{Le rôle déterminant des éléments conditionnels}

L'identification des bénéfices et des coûts liés à l'utilisation d'applications mobiles n'a de sens que si l'on prend en considération les éléments conditionnels. En accord avec les travaux de Sheth, et al. (1991), Gummerus et Pihlström (2011) et Wang et al. (2013), nous présentons les éléments conditionnels pouvant affecter la valeur globale de l'usage d'une application mobile en magasin.

La personnalisation est le premier élément conditionnel relevé sur l'application push et idéale. A propos de l'application idéale, la personnalisation est l'expression d'une volonté générale de pouvoir construire celle-ci en fonction de ses goûts (Gummerus et Pihlström, 2011; Sheth et 
al., 1991; Wang et al., 2013) ("Moi j'aimerais qu'on puisse la personnaliser comme on le souhaite. Totalement. Hyper personnalisable. »(H18)). Tandis que pour l'application push, la personnalisation joue un autre rôle dans l'acceptation. Rappelons que l'application push envoie des notifications à tout moment. Si ces notifications étaient personnalisables, elles seraient plus acceptables ("Après si on peut filtrer les messages qu'on reçoit. Si on reçoit par exemple que les messages de promos...Ce dont on a envie vraiment. Il faut qu'on sélectionne les catégories » (F18)) et les utilisateurs accepteraient d'être suivis ("Je veux bien être pistée mais ...»(F40)). Plus globalement, on relève les mêmes volontés concernant la personnalisation globale de l'application que celles relevées pour l'application idéale (" Quelque chose encore plus, quelque chose fait spécialement pour ça. Je préfère que tout soit centralisé sur mon téléphone que je peux personnaliser, plutôt qu'on m'impose quelque chose avec un caddie. Là vous dites "non je ne veux pas cette application » mais quand on vous dit sur le caddie que tout le monde va avoir vous dites oui. »(H19)).

Par ailleurs, le second élément conditionnel est étroitement lié à la personnalisation et plus particulièrement à la nécessite de recevoir des offres de réductions. Quand le contexte économique est fragile et que le pouvoir d'achat baisse, cela conduit à la recherche d'économies ("Après, si c'est intelligent, et qu'on propose des réductions, des promotions... ça permet de faire des économies, tu vois, des bons de réductions ou autres en passant par cette application oui... »(F46)), pour l'application pull. Alors que l'opportunité de faire de bonnes affaires est recherchée pour l'application push ("Après, si tu tombes sur quelque chose avec une bonne promo, que tu ne connais pas, ça peut être intéressant. » (F18)).

Finalement, on relève deux autres éléments conditionnels pour l'application pull, qui pourraient être transposables aux autres types d'applications. S'agissant en premier lieu du type de magasin, on remarque une nette préférence chez les interviewés pour certains contextes d'utilisation de l'application. En effet, certains types de point de vente se prêteraient mieux à l'utilisation de l'application comme par exemple les magasins de prêt à porter (" Oui c'est plus dans ce style de magasins " (H19)). Les magasins aux achats plus hédoniques sont davantage susceptibles de rendre l'utilisation de l'application intéressante ("Pas au quotidien. Juste pour des objets. » $(F(1) 19))$. En effet, les clients ont plus de temps pour effectuer leur achat du fait que celui-ci est plus réfléchi ("Si c'est un gros objet à acheter oui. » (F18)). Les magasins de prêt à porter, par exemple, entrent dans ce cas de figure. A l'inverse, les magasins alimentaires ne suscitent pas l'envie d'utiliser l'application. Les achats sont plus courants et automatiques, et l'acte d'achat est considéré comme moins hédonique. ("Ça dépend si l'objet ne le justifie pas. Si c'est un tout petit truc... je ne sais pas moi si j'ai juste oublié d'acheter des æufs. Je ne sais pas. Il y a des choses pour lesquelles c'est justifiable mais ... »(H18)).

Enfin, la forte affluence en point de vente est une situation pouvant influencer l'utilisation (paiement via l'application) de l'application pull ("Ben je pense que vraiment dans la seule situation ou je pourrais utiliser un truc comme ça ... je pense que ce serait vraiment en période de solde où il y a une foule... Ou j'ai juste envie de quitter le magasin » (F25)). Si l'attente à la caisse est longue, cela peut favoriser l'utilisation de l'application ("Voilà juste la situation où on pourrait utiliser cette appli c'est comme il le disait au départ dans la vidéo, s'il y a une queue immense et que t'as la flemme »(H22)).

\section{Conclusion}

Ce travail avait pour ambition d'identifier les sources de création (bénéfices) et de destruction (coûts) de valeur pour le client lorsqu'il utilise une application mobile d'aide à l'achat en magasin. Nous avons exposé les différentes dimensions relevées par type d'application. D'un point de vue théorique, nous avons confirmé un certain nombre de bénéfices « classiques » 
ayant déjà été relevés dans des travaux antérieurs, mais aussi des dimensions plus spécifiques aux applications d'aide à l'achat comme, par exemple, "l'organisation ». 
Tableau 1 : Comparaison de notre approche avec les études antérieures

\begin{tabular}{|c|c|c|c|c|c|}
\hline Approche & Dimensions & Type d'étude & $\begin{array}{l}\text { Variables } \\
\text { conditionnelles }\end{array}$ & $\begin{array}{l}\text { Contexte d'étude et/ou type } \\
\text { d'application }\end{array}$ & Auteurs \\
\hline $\begin{array}{l}\text { Approche hybride } \\
\text { inspirée du TAM }\end{array}$ & $\begin{array}{l}\text { Bénéfices : qualité de l'information, qualité du } \\
\text { service, qualité du système } \\
\text { Sacrifices : effort technologique, coûts } \\
\text { d'utilisation perçus, risques perçus }\end{array}$ & $\begin{array}{l}\text { Étude quantitative sur } 235 \\
\text { personnes ayant eu une } \\
\text { expérience avec un téléphone } \\
\text { mobile }\end{array}$ & & $\begin{array}{l}\text { Application de réservation } \\
\text { d'hôtels }\end{array}$ & $\begin{array}{l}\text { (Hsiu-Yuan Wang and } \\
\text { Wang 2010) }\end{array}$ \\
\hline $\begin{array}{l}\text { Approche hybride } \\
\text { de la valeur }\end{array}$ & $\begin{array}{l}\text { Bénéfices : commodité temporelle, contrôle du } \\
\text { service, compatibilité du service } \\
\text { Coûts : risques, effort cognitif }\end{array}$ & $\begin{array}{l}\text { Étude quantitative auprès de } \\
375 \text { répondants détenteurs } \\
\text { d'un téléphone mobile et } \\
\text { familiers des nouvelles } \\
\text { technologies }\end{array}$ & Conscience du temps & Application mobile de service & $\begin{array}{l}\text { (Kleijnen, de Ruyter, } \\
\text { and Wetzels 2007) }\end{array}$ \\
\hline $\begin{array}{l}\text { Approche hybride } \\
\text { de la valeur }\end{array}$ & $\begin{array}{l}\text { Valeur : utilitaire, hédonique, sociale } \\
\text { Coûts : risque lié au temps, risque social, risque } \\
\text { psychologique, risque lié à la vie privée }\end{array}$ & $\begin{array}{l}\text { Étude quantitative sur } 230 \\
\text { individus utilisateurs de } \\
\text { smartphone et propriétaire } \\
\text { d'une carte de crédit }\end{array}$ & & Adoption du paiement mobile & $\begin{array}{l}\text { (Cocosila and Trabelsi } \\
\text { 2016) }\end{array}$ \\
\hline Adaptation du TAM & $\begin{array}{l}\text { Valeur : fonctionnelle, sociale, émotionnelle, } \\
\text { épistémique }\end{array}$ & $\begin{array}{l}\text { Étude quantitative auprès de } \\
282 \text { utilisateurs d'applications } \\
\text { mobiles }\end{array}$ & Valeur conditionnelle & Applications mobiles & $\begin{array}{l}\text { (Hsiu-Yu Wang, Liao, } \\
\text { and Yang 2013) }\end{array}$ \\
\hline Adaptation du TAM & $\begin{array}{l}\text { Facilité d'utilisation perçue, utilité perçue, praticité, } \\
\text { plaisir }\end{array}$ & $\begin{array}{l}\text { Étude quantitative auprès de } \\
474 \text { utilisateurs d'applications } \\
\text { mobiles de m-commerce }\end{array}$ & $\begin{array}{l}\text { Customisation de } \\
\text { l'application mobile }\end{array}$ & Application de M-commerce & (McLean 2018) \\
\hline Adaptation du TAM & $\begin{array}{l}\text { Commodité temporelle, interactivité, compatibilité, } \\
\text { effort attendu }\end{array}$ & $\begin{array}{l}\text { Étude quantitative sur } 503 \\
\text { utilisateurs d'applications } \\
\text { mobiles }\end{array}$ & & $\begin{array}{l}\text { Diverses applications utilisables } \\
\text { dans une multitude de contextes } \\
\text { (e.g. facebook, Google, etc.) }\end{array}$ & (Kim and Baek 2018) \\
\hline $\begin{array}{l}\text { Approche } \\
\text { multidimensionnelle } \\
\text { de la valeur }\end{array}$ & $\begin{array}{l}\text { Valeur : optimisation de choix, situationnelle, } \\
\text { utilitaire (économique, informationnelle et de } \\
\text { commodité), hédonique (esthétique, réassurance, } \\
\text { récréative) et valeur de lien social }\end{array}$ & $\begin{array}{l}\text { Étude qualitative menée } \\
\text { auprès de } 10 \text { femmes et } 20 \\
\text { hommes }\end{array}$ & & $\begin{array}{l}\text { Usage des applications mobiles } \\
\text { diverses et variées (e.g. } \\
\text { Groupon, ventes privées) }\end{array}$ & $\begin{array}{l}\text { (Gonzalez, Huré, and } \\
\text { Picot-Coupey 2012) }\end{array}$ \\
\hline $\begin{array}{l}\text { Approche hybride } \\
\text { de la valeur }\end{array}$ & $\begin{array}{l}\text { Bénéfices : praticité, efficacité, meilleure } \\
\text { organisation, hédonique, contrôle, soi social, social. } \\
\text { Coûts : invasion, atteinte à la vie privée, relationnel, } \\
\text { éthique, perte de plaisir d'achat, émotionnel, } \\
\text { dépendance, statut, perte de contrôle. }\end{array}$ & $\begin{array}{l}\text { Étude qualitative menée avec } \\
\text { trois focus groups }\end{array}$ & $\begin{array}{l}\text { Pouvoir d'achat, type de } \\
\text { magasin, affluence, } \\
\text { personnalisation. }\end{array}$ & $\begin{array}{l}\text { Distinction entre les } \\
\text { applications utilisées au point } \\
\text { de vente physique et les } \\
\text { application M-commerce. } \\
\text { Distinction des types } \\
\text { d'applications utilisées au point } \\
\text { de vente physique. }\end{array}$ & Notre approche \\
\hline
\end{tabular}


L'apport le plus conséquent se situe dans l'identification des coûts liés à l'usage. Nous avions souligné précédemment le manque de prise en compte, dans les travaux existants, de nouveaux coûts ayant émergés avec le digital (Kumar et Reinartz, 2016). Cette recherche vient confirmer également les propos de Newman et al. (2017) et Collier et al. (2014), sur la différence de perception des SST par les clients. Effectivement, certains coûts identifiés (invasion, perte de plaisir d'achat, dépendance, soi social et atteinte à la vie privée) présentent une vraie différence comparés à ceux identifiés sur d'autres SST (Mencarelli et Rivière, 2014) ${ }^{5}$. Par ailleurs, cette différence est aussi valable pour la nature de l'application, dont l'identification constitue le deuxième objectif de ce papier. En effet, on remarque clairement des différences de perception des sources de valeur en fonction du type d'application. L'application pour les achats en ligne présente, par exemple, moins de coûts que celle utilisée en magasin. Le contexte du point de vente fait ressortir des coûts inexistants dans le canal virtuel, du fait de la relation client-vendeur.

Enfin, les éléments conditionnels identifiés ont permis de confirmer les travaux de Sheth et al. (1991), Gummerus et Pihlström (2011) et Wang et al. (2013) quant à leur contribution dans la construction de la valeur globale. Notons que ces éléments sont spécifiques au point de vente physique et à la nature de l'application. Cette recherche argumente alors en faveur de la prise en compte des éléments conditionnels dans l'appréciation de la valeur globale du service mobile.

Sur le plan managérial, nous avons pu mettre en évidence les leviers participant à la construction et à la destruction de la valeur perçue d'une application mobile, à l'aide de techniques projectives. La faible utilisation, jusqu'à ce jour, d'applications mobiles en point de vente, nous a conduit vers cette technique portant sur des applications réalistes (une déjà existante et l'autre émergente). L'identification des sources de valeur permet aux distributeurs de repérer les bénéfices potentiels pouvant être associés à leur application dès leur phase de développement. Une meilleure connaissance des coûts potentiels comme par exemple l'atteinte à la vie privée, l'invasion et le coût social de l'application, permettrait également aux distributeurs de travailler en amont sur ces leviers afin d'en limiter l'impact négatif potentiel. L'éclairage sur les éléments conditionnels donne aussi des indications sur les meilleures conditions d'utilisation des applications mobiles. L'analyse des dimensions de bénéfices et de coûts relevées, couplée aux éléments conditionnels, plaide pour une utilisation des applications dans le cadre d'achats plus impliquants et dans des points de vente non alimentaires. Néanmoins, l'usage des applications mobiles dans les points de vente alimentaires pourrait aussi être favorisé dans la mesure où l'application répondrait à la recherche d'économies que verbalisent les interviewés.

A la lumière de ces constats et en accord avec les travaux antérieurs, nous pouvons apporter quelques pistes de réflexion sur les applications d'aide à l'achat au point de vente. Dans la continuité des travaux sur les SST de Lapassouse-Madrid et Vlad (2016), le développement d'applications mobiles doit s'opérer dans le respect de la relation client-vendeur, en visant la complémentarité. En dehors de ces relations humaines, notre recherche sensibilise également sur le respect de la relation client-produit. Le service mobile ne doit pas se dresser en obstacle au toucher et à la manipulation du produit et faire disparaitre la partie hédonique de l'achat. Enfin, nous nous inscrivons dans les recommandations faites par Mencarelli et Rivière (2014) sur la nécessité d'avoir une politique de communication lors de la mise en place de services digitaux. Ceci, afin de réduire les coûts potentiels qui pourraient être perçus mais également pour rassurer les clients sur le respect de la relation client-vendeur et la préservation des emplois. Globalement, la mise en place de ce type de dispositif technologique constitue un grand intérêt

\footnotetext{
${ }^{5}$ Mencarelli et Rivière (2014) identifient les sacrifices suivants chez les SST: coût monétaire, coût fonctionnel, risque perçu, coût psychologique, coût temporel, coût éthique, coût relationnel, coût informationnel et coût d'équité.
} 
pour les distributeurs (Goudey, 2013), mais une attention particulière doit être portée aux points de vigilance cités précédemment.

Les contributions de ce travail doivent être appréciées en tenant compte de ses limites. S'agissant d'une étude exploratoire mobilisant des focus-groups, les avis et sentiments exprimés ne représentent pas la pensée générale de tous les individus. En outre, la variété des applications étudiées dans cette recherche ne reflète pas la complète diversité des applications existantes. Un travail plus approfondi, dans un contexte concret de point de vente, pourrait apporter davantage de connaissances sur l'appréciation des sources de valeur. Plus généralement, les recherches gagneraient en précision, à se focaliser sur l'étude de l'usage des applications dans des contextes et produits/services variés, sur différentes applications et dans diverses conditions d'utilisation (e.g. forte affluence, période creuse). En dernier lieu, il nous semble important de souligner une limite concernant le périmètre de ce travail de recherche qui ne prend pas en compte les sites mobiles. Dans une perspective de recherche future, il serait pertinent de comparer les valeurs perçues entre l'application mobile proposée par le distributeur et les sites mobiles offrant des services similaires. En effet, l'utilisateur doit souvent faire le choix entre ces deux canaux d'informations qui présentent des différences significatives.

\section{Bibliographie}

AURIER P, EVRARD Y, \& N'GOALA G. (2004). Comprendre et mesurer la valeur du point de vue du consommateur. Recherche et Applications en Marketing, Vol. 19, No. 3, p. 1-20.

BABIN B J, DARDEN W R, \& GRIFFIN M. (1994). Work and/or Fun: Measuring Hedonic and Utilitarian Shopping Value. Journal of Consumer Research, Vol. 20, No. 4, p. p. 644-656.

BOUZID Y, \& VANHEEMS R. (2014). Comportement web-to-store : vers une nouvelle logique de contrôle de soi ? Management \& Avenir, Vol. 71, No. 5, p. 189.

DAVIS F D. (1989). Perceived Usefulness, Perceived Ease of Use, and User Acceptance of Information Technology. MIS Quarterly, Vol. 13, No. 3, p. p. 319-340.

DE RUYTER K, WETZELS M, LEMMINK J, \& MATTSON J. (1997). The dynamics of the service delivery process: a value-based approach. International journal of research in marketing, Vol. 14, No. 3, p. $231-243$. DODDS W B, \& MONROE K B. (1985). The Effect of Brand and Price Information on Subjective Product Evaluations. Advances in Consumer Research, Vol. 12, No. 1, p. 85-90.

FEENSTRA F, \& GLÉRANT-GLIKSON A. (2017). Identifier et comprendre les sources de valeur dans l'interaction avec les SSIT (Self-Service Information Technologies) en magasin. Décisions Marketing, Vol. 86, p. 47-66.

GONZALEZ C, HURÉ E, \& PICOT-COUPEY K. (2012). Usages et valeur des applications mobiles pour les consommateurs: quelles implications pour les distributeurs? In Actes du 15 e Colloque international E. Thil, Lille, France.

GOUDEY A. (2013). Exploration des effets du degré de technologie perçu du magasin sur le comportement de magasinage. Management \& Avenir, Vol. 63, No. 5, p. 15.

GROß M. (2015). Exploring the acceptance of technology for mobile shopping: an empirical investigation among Smartphone users. The International Review of Retail, Distribution and Consumer Research, Vol. 25, No. 3, p. 215-235.

GUMMERUS J, \& PIHLSTRÖM M. (2011). Context and mobile services' value-in-use. Journal of Retailing and Consumer Services, Vol. 18, No. 6, p. 521-533.

HA L. (1996). Observations: Advertising Clutter in Consumer Magazines: Dimensions and Effects. Journal of Advertising Research, Vol. 36, No. 4, p. 76-84.

HIRSCHMAN E C, \& HOLBROOK M B. (1982). Hedonic Consumption: Emerging Concepts, Methods and Propositions. Journal of Marketing, Vol. 46, p. p. 92-102, a.

HOLBROOK M B, \& CORFMAN K P. (1985). Quality and Value in the Consumption Experience: Phaedrus Rides Again. Perceived quality, Vol. 31, No. 2, p. p. 31-57.

KALLWEIT K, SPREER P, \& TOPOROWSKI W. (2014). Why do customers use self-service information technologies in retail? The mediating effect of perceived service quality. Journal of Retailing and Consumer Services, Vol. 21, No. 3, p. 268-276, mai.

KANG J-Y M, MUN J M, \& JOHNSON K K P. (2015). In-store mobile usage: Downloading and usage intention toward mobile location-based retail apps. Computers in Human Behavior, Vol. 46, p. 210-217. KIM B, \& HAN I. (2011). The role of utilitarian and hedonic values and their antecedents in a mobile data 
service environment. Expert Systems with Applications, Vol. 38, No. 3, p. 2311-2318.

KIM B, \& OH J. (2011). The difference of determinants of acceptance and continuance of mobile data services: A value perspective. Expert Systems with Applications, Vol. 38, No. 3, p. 1798-1804.

KLEIJNEN M, DE RUYTER K, \& WETZELS M. (2007). An assessment of value creation in mobile service delivery and the moderating role of time consciousness. Journal of Retailing, Vol. 83, No. 1, p. 33-46.

KUMAR V, \& REINARTZ W. (2016). Creating Enduring Customer Value. Journal of Marketing, Vol. 80, No. 6, p. 36-68, November.

LAI A W. (1995). Consumer Values, Product Benefits and Customer Value: A Consumption Behavior Approach. Advances in Consumer Research, Vol. 22, No. 1, p. 381-388.

LANCELOT-MILTGEN C, \& LEMOINE J-F. (2015). Mieux collecter les données personnelles sur Internet. Une étude qualitative auprès d'internautes français. Décisions Marketing, Vol. 79, p. 35-52.

LAPASSOUSE-MADRID, C, \& VLAD M. (2016). Courses connectées : un cas de destruction ou de création de valeur pour les clients et les distributeurs. Décisions Marketing, No. 84, p. 17p.

MATHWICK C, MALHOTRA N, \& RIGDON E. (2001). Experiential value: conceptualization, measurement and application in the catalog and Internet shopping environment. Journal of Retailing, No. 77, p. p. 39-56. MENCARELLI R, \& RIVIËRE A. (2014). La participation du client dans un contexte de self-service technologies. Une approche par la valeur perçue. Revue française de gestion, Vol. 40, No. 241, p. 13-30. MERISAVO M, KAJALO S, KARJALUOTO H, VIRTANEN V, SALMENKIVI S, RAULAS M, \& LEPPÄNIEMI M. (2007). An Empirical Study of the Drivers of Consumer Acceptance of Mobile Advertising. Journal of Interactive Advertising, Vol. 7, No. 2, p. 41-50.

MUKAMURERA J, LACOURSE F, \& COUTURIER Y. (2006). Des avancées en analyse qualitative: pour une transparence et une systématisation des pratiques. Recherches qualitatives, Vol. 26, No. 1, p. 110-138.

NEWMAN C L, WACHTER K, \& WHITE A. (2017). Bricks or clicks? Understanding consumer usage of retail mobile apps. Journal of Services Marketing.

NYSVEEN H, PEDERSEN P E, \& THORBJØRNSEN H. (2005). Intentions to use mobile services: Antecedents and cross-service comparisons. Journal of the Academy of Marketing Science, Vol. 33, No. 3, p. 330.

OZTURK A B, NUSAIR K, OKUMUS F, \& HUA N. (2016). The role of utilitarian and hedonic values on users' continued usage intention in a mobile hotel booking environment. International Journal of Hospitality Management, Vol. 57, p. 106-115.

REVELS J, TOJIB D, \& TSARENKO Y. (2010). Understanding consumer intention to use mobile services. Australasian Marketing Journal (AMJ), Vol. 18, No. 2, p. 74-80.

RIVIĖRE A, \& MENCARELLI R. (2012). Vers une clarification théorique de la notion de valeur perçue en marketing. Recherche et Applications en Marketing, Vol. 27, No. 3, p. 97-123.

ROSENBERG M. (1979). Conceiving the self. Basic Books.

RUIZ D M, GREMLER D D, WASHBURN J H, \& CARRIÓN G C. (2008). Service value revisited: Specifying a higher-order, formative measure. Journal of Business Research, Vol. 61, No. 12, p. 1278-1291.

SHETH J N, NEWMAN B I, \& GROSS B L. (1991). Why we buy what we buy: A theory of consumption values. Journal of Business Research, Vol. 22, No. 2, p. 159-170.

SPIGGLE S. (1994). Analysis and Interpretation of Qualitative Data in Consumer Research. Journal of Consumer Research, Vol. 21, No. 3, p. 491-503.

SWEENEY J C, \& SOUTAR G N. (2001). Consumer perceived value: The development of a multiple item scale. Journal of retailing, Vol. 77, No. 2, p. 203-220.

TOJIB D, \& TSARENKO Y. (2012). Post-adoption modeling of advanced mobile service use. Journal of Business Research, Vol. 65, No. 7, p. 922-928.

VERNETTE E. (2008). «Les atouts et les pièges de la personnalité de marque ». Décisions Marketing, No. 49, p. 19-31.

VESPE F. (1997). High-Tech Billboards: The Same Old Litter on a Stick. Journal of Public Policy \& Marketing, Vol. 16, No. 1, p. 176-179.

WANG H-Y, LIAO C, \& YANG L-H. (2013). What Affects Mobile Application Use? The Roles of Consumption Values. International Journal of Marketing Studies, Vol. 5, No. 2.

ZEITHAML V A. (1988). Consumer Perceptions of Price, Quality, and Value: A Means-End Model and Synthesis of Evidence. Journal of Marketing, Vol. 52, No. 3, p. p. 2-22. 